\title{
Monte Carlo Simulation of a Position Sensitive Gamma Ray Detector
}

\author{
Marcelo de Almeida and Maurício Moralles \\ Centro do Reator de Pesquisas, Instituto de Pesquisas Energéticas e Nucleares, \\ Caixa Postal 11049, CEP 05422-970, São Paulo, SP, Brazil
}

\section{Received on 13 November, 2004}

\begin{abstract}
The Monte Carlo method was employed to obtain the response of a position sensitive gamma ray detector. The basic unit consists of a scintillator bar coupled to two photon detectors. Determination of the position of interaction is obtained from the ratio of the light intensity measured at each extremity of the bar. Association of two or more detectors of this type can be used to build a system with potential use in positron emission tomography. For practical purposes, a CsI(Tl) bar with dimensions of $15 \times 1 \times 1 \mathrm{~cm}^{3}$ was considered. Simulations were performed with the GEANT4 toolkit, using the physical processes of low-energy electromagnetic extension. Light attenuation coefficient and energy resolution of $\mathrm{CsI}(\mathrm{Tl})$ bars were included according to data found in the literature. Results obtained from detector irradiation with $511 \mathrm{keV}$ annihilation photons indicate that the position of interaction can be determined with uncertainty of $3 \mathrm{~mm}$. Simulations of positron sources placed between two detectors were also performed and two dimensional images were obtained.
\end{abstract}

\section{INTRODUCTION}

Nowadays positron emission tomography (PET) systems are widely used in nuclear diagnostic medicine. In this imaging technique positron-emitting isotopes are introduced into the patient, and the two $511 \mathrm{keV}$ photons emitted following electron-positron annihilation are observed in coincidence. Typical PET scanners have many detection basic units, formed by several small scintillator crystals coupled to positionsensitive photomultiplier tubes [1]. The aim of this work is to study a new concept of PET systems, in which the basic unit consists of a small CsI(Tl) scintillator bar coupled to two photodiodes in its extremities. With this type of basic unit, it would be possible to build a low-cost compact PET system.

This study has been developed through computer simulations using the Monte Carlo method, implemented with the GEANT4 toolkit [2]. CsI(Tl) crystal was chosen because its emission spectrum have the maximum at $550 \mathrm{~nm}$, which is suitable for photodiode readout. Recent developments on the growth of $\mathrm{CsI}(\mathrm{Tl})$ crystals allows the production of this scintillator with low afterglow, which makes it better suited for tomography applications [3]. Parameters for the CsI(Tl) bars were extracted from the experimental work of Labanti et al. [4].

\section{SIMULATION OF BASIC DETECTION UNIT}

The detector simulation was performed by using the lowenergy electromagnetic extension of the GEANT4 toolkit. GEANT4 is a package of $\mathrm{C}++$ classes that allows the development of codes for Monte Carlo calculations which involves the interaction of radiation with matter. Recent versions of GEANT4 have a low-energy extension for electromagnetic processes, which was validated for use in medical physics applications [5].

In this work, the considered interactions were Rayleigh scattering, Compton scattering, photoelectric effect, ionization and bremsstrahlung. The simulated basic detector unit consisted of a small CsI bar of dimensions $15 \times 1 \times 1 \mathrm{~cm}^{3}$ cou- pled to two photodiodes at the scintillator bar extremities (Fig. 1). The chosen dimensions were based in the experimental work of Labanti et al. [4], from where we extracted parameters for the modelling of the optical and electronic response of this detector. Since Tl concentrations are very low in real scintillators, assuming the bar material as pure CsI becomes a good approximation for the simulation purpose.

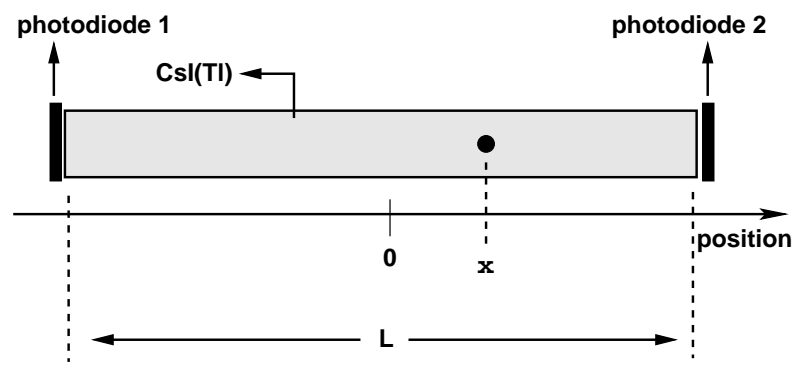

FIG. 1: Scheme of the basic detection unit.

The light intensity observed by each photodiode corresponds to a fraction of the light emitted by the radiation interaction, attenuated along the bar until it reaches the photodiode. According to the scheme shown in Fig. 1, the signals $E_{1}$ and $E_{2}$, of the left and right photodiodes respectively, are calculated through

$$
E_{1}=\frac{K E_{0}}{2} \exp \left(-\alpha\left(\frac{L}{2}+x\right)\right)
$$

and

$$
E_{2}=\frac{K E_{0}}{2} \exp \left(-\alpha\left(\frac{L}{2}-x\right)\right)
$$

where $K$ is a constant, $E_{0}$ is the energy deposited in detector, $L$ is the crystal bar length and $\alpha$ is the light attenuation coefficient. The value of $\alpha=0.099 \mathrm{~cm}^{-1}$, was taken from the results of ref. [4]. 


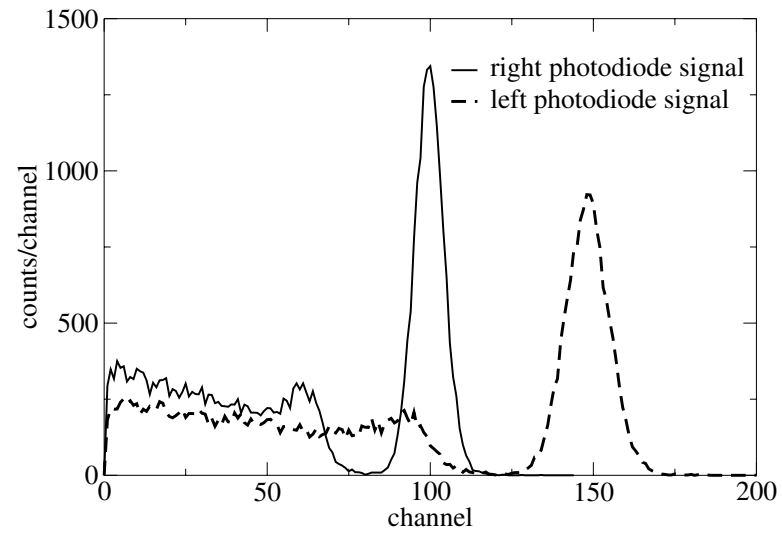

FIG. 2: Photodiode signals for incidence position of $5.5 \mathrm{~cm}$ relative to the left extremity of the bar.

The position of interaction are determined from the signals of the photodiodes through the expression [6]

$$
x=\frac{1}{2 \alpha} \ln \frac{E_{2}}{E_{1}} .
$$

The position sensitivity was obtained by simulating a well collimated source of $511 \mathrm{keV}$ gamma rays placed in different positions. The gamma rays were directed perpendicular to the surface of the bar at different distances relative to the extremities. A Gaussian dispersion corresponding to an energy resolution of $10 \%$ (FWHM) was applied to the photodiode readout signals $\left(E_{1}\right.$ and $\left.E_{2}\right)$. This corresponds to characteristic energy resolution for CsI(Tl) [6]. Fig. 2 shows spectra of the signals of each photodiode for incidence position of $5.5 \mathrm{~cm}$ relative to the left extremity of the bar.

The position was calculated for several incident points and its uncertainty was determined as $0.32 \mathrm{~cm}$ in the central region of the bar, excluding $2.5 \mathrm{~cm}$ of both extremities. This result is in agreement with the experimental one [4]. Deterioration of the energy resolution was observed when the interactions occur at distances of less than $2.5 \mathrm{~cm}$ from the extremities of the detector bar.

\section{TWO DETECTORS SYSTEM}

In order to evaluate a system that allows the determination of the position of a positron source in space, an arrangement composed by two parallel detectors at a distance of $15 \mathrm{~cm}$ was simulated as shown in Fig. 3. The source was positioned between the detector bars, and emitted two $511 \mathrm{keV}$ photons in opposite sense, with direction given by a random angle in the range of 360 degrees.

The simulation provided the signals from the photodiodes readout, and the position of interaction in both crystal bars were calculated for each event. We assume one event as the set of interactions and performed calculations from the radiation emission till the readout of the photodiodes. One event provides two pairs of numbers, each pair corresponding to the photodiode readout of one bar.
Three validation conditions were applied to the data. Every event which did not satisfy one of those conditions was excluded. At first, all events with no signal in one of the detector bars were excluded, which means that there was no interaction in one of the bars. The second condition validated the interaction position: all events with interaction position determined as less than $2.5 \mathrm{~cm}$ from any detector extremity were considered not valid, because of the resolution deterioration mentioned above. This condition limited the usable region to $10 \mathrm{~cm}$ centered at the midpoint of the bar. Finally, the third condition eliminated events with partial absorption of the $511 \mathrm{keV}$ energy. This condition excluded Compton scattered photons in either of the crystal bars. It is well known that Compton scattering in the detectors and in the environment can produce wrong determination of the source position. Fig. 4 shows that when total absorption of the $511 \mathrm{keV}$ photons occurs in the crystal bar, the sum of the signals of both photodiodes is at least in channel 200. This value was used as threshold for the third validation condition.

\section{VISUALIZATION OF THE SYSTEM RESPONSE}

A simple algorithm was used to visualize the response of the system, which was performed by summing lines in a discrete plane represented by a matrix. For each event one straight line was plotted from the determined positions of interactions in the detector bars, as shown in Fig. 5. Counts are added to the cells of the matrix that are crossed by the line. After several events, in the case where there is only one source, the cell with the largest number of counts corresponds to the source position.

An example with five sources is shown in the surface plot of Fig. 6 . In this case the grid has $20 \times 20$ cells (each cell has $5 \times$ $5 \mathrm{~mm}^{2}$ ), the sources have the same activity and were placed in positions corresponding to cells at $(5,5),(5,15),(10,10)$, $(15,5)$ and $(15,15)$. Although this method of visualization is not suitable to determine the distribution of sources in a realistic case of medical imaging, peaks are observed in Fig. 6 , corresponding to the five source positions.

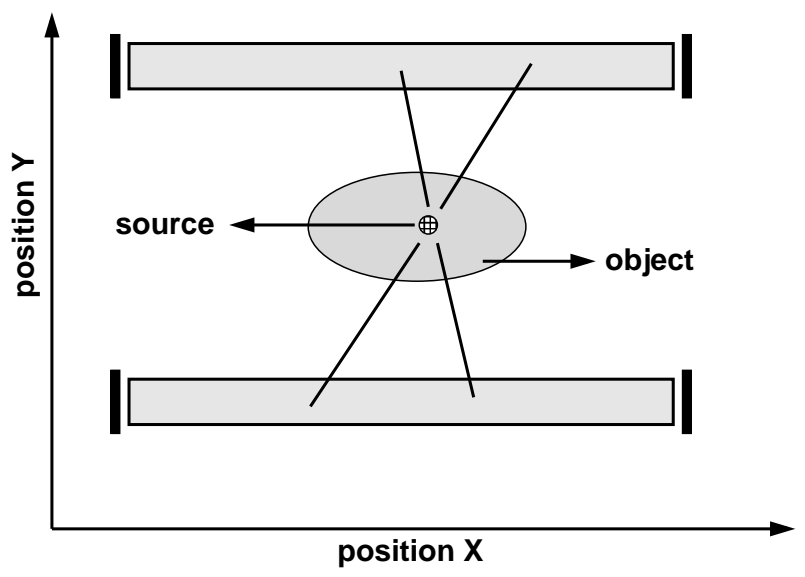

FIG. 3: Scheme of the two detectors system. 


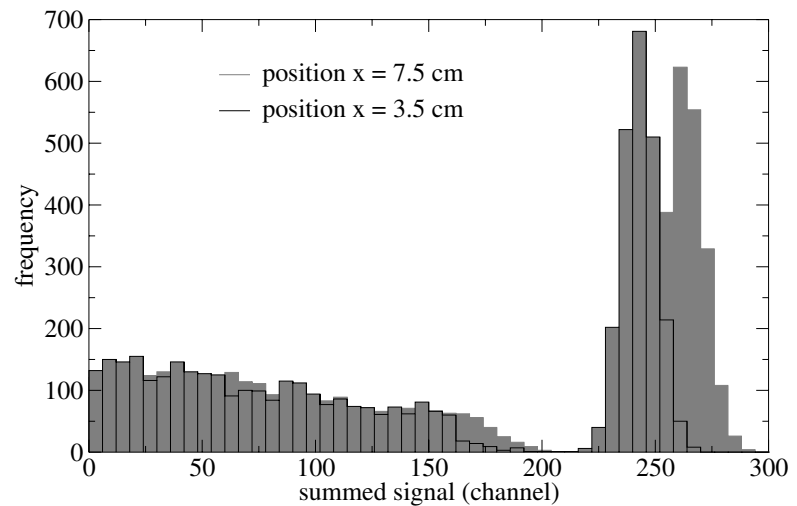

FIG. 4: Summed signal $\left(E_{1}+E_{2}\right)$ for incidence positions of 3.5 and $7.5 \mathrm{~cm}$ relative to the left extremity of the bar.

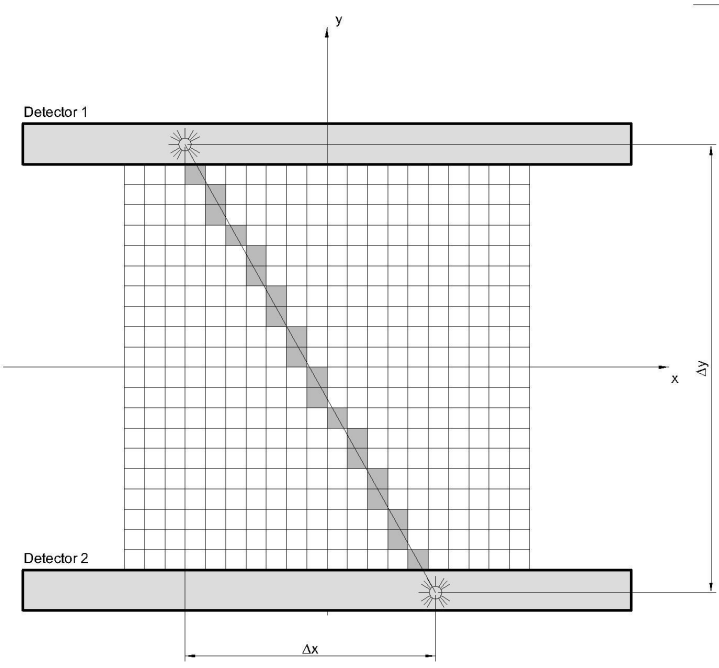

FIG. 5: Representation of a line defined by one event in the discrete plane between the two detectors.

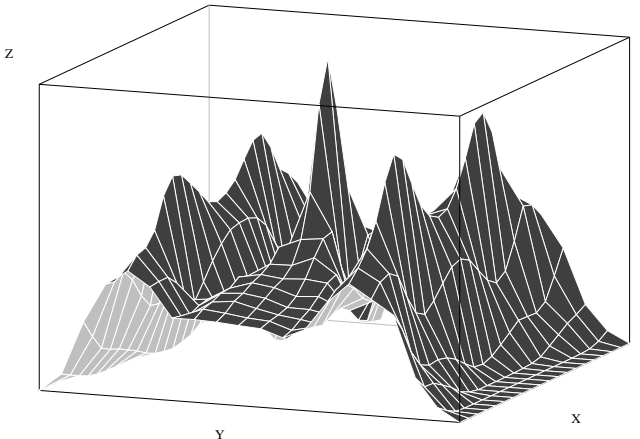

FIG. 6: Surface plot of the summed lines for five sources.

\section{CONCLUSION}

This work presents a successfully application of GEANT4 low-energy extension in a problem of medical physics interest. The simulation of the studied position sensitive detector was in agreement with experimental results and provided support for an application in tomography. The visualization of the system response is very limited for medical imaging purpose, although the results encourage development of more sophisticated method for image reconstruction.

\section{Acknowledgments}

This research was supported by $\mathrm{CNPq}$ (Conselho Nacional de Desenvolvimento Cientfico e Tecnolgico - Brasil).
[1] W.W. Moses, Nucl. Instr. and Meth. A 471, 209 (2001).

[2] S. Agostinelli et al., Nucl. Instr. and Meth. A 506, 250 (2003).

[3] Products catalogue of Moltech GmbH. Website http://www.mtberlin.com

[4] C. Labanti et al, Nucl. Instr. and Meth. A 310, 327 (1991).
[5] J-F. Carrier, L. Archambault, L. Beaulieu, Med. Phys. 31, 484 (2004).

[6] G.F. Knoll. Radiation Detection and Measurement. John Wiley and Sons, Inc. New York, 1989. 